\title{
Divulgación de principios del Urbanismo Contemporáneo: Aplicación a Magallanes (1936)
}

Luis Muñoz Maluschka (1896-1974)

\section{Filiación}

Arquitecto y Urbanista chileno (1896-1974), Jefe de la Sección de Urbanismo del M.O.P. Chile (c. 1936 hasta 1952), profesor de Urbanismo de la Escuela de Arquitectura de la Facultad de Arquitectura de la Universidad de Chile entre 1947-52. Participó de manera fundamental en la creación de las leyes que crearon en 1953, la CORVI, la Dirección de Planeamiento, y la ley que definió los niveles territoriales de la Planificación Urbana sobre el nivel comunal, en Chile.

\section{Resumen}

Siempre que el aumento de la velocidad de los medios de transporte fuera proporcional con el aumento de la riqueza territorial-medida en sus índices de extracción y elaboración-, se mantendría el equilibrio en las funciones económicas de los núcleos poblados, pero hasta la fecha, [1936] y particularmente en territorios de colonización, el aumento de la velocidad ha sido muy superior al incremento de la riqueza territorial. Las previsiones y consecuencias de este fenómeno de concentración de funciones económicas, debe tomarse en cuenta en el caso de Magallanes (Chile) y se deducen de un análisis de las posibilidades de incremento de la riqueza y del mejoramiento de la vialidad territorial.

\section{Palabras clave}

Ordenación del territorio, áreas de colonización, Magallanes - Chile, Luis Muñoz Maluschka.

\begin{abstract}
If the increase in the speed of transportation was proportional to the increase in the land value, measured by the levels of exploitation and manufacturing, there would be an equilibrium in the economical functions within the towns. But up to date [1936] and particular in colonization areas the increase in speed has been greater than the increase of the land value. The expectations and consequences of this concentration of economical functions phenomenon should be taken in account while reviewing the particular case of Magallanes (Chile). The possible increase of the land value and the need for improvement of the roads should be analyzed.
\end{abstract}

\section{Key words}

Land arrangement, colonization territory, Magallanes - Chile, Luis Muñoz Maluschka. 


\section{Sumario}

Introducción

1.-La zonificación de los sectores urbanos

2.-Futura extensión y crecimiento

3.-Aspectos de la vida vegetativa

4.-Otras actividades urbanas

\section{Introducción}

El urbanismo como arte o técnica existe desde que la humanidad comenzó a vivir en grandes aglomeraciones.

Evoquemos la belleza incomparable de la Acrópolis, de Pompeya, de las ruinas reconstruidas de ciudades faraónicas, o de la colonización greco-romana en otros países, para que tengamos que comprender que esa belleza no ha sido obra de la casualidad, sino la creación del artista realizada por intermedio de un plan verificado a través de las generaciones.

Las ciudades renacentistas fueron formadas de acuerdo con planes que buscaban efectos decorativos o plásticos, emplazamientos monumentales de los edificios o palacios que levantara el régimen monárquico.

En las ciudades del pasado el artista buscaba efectos de plástica, plazas proporcionadas a la altura y volumen de los edificios antepuestos.

Las necesidades de tránsito, quedaban reducidas a la tracción animal y al movimiento de peatones, y otras necesidades casi no eran consideradas.

El hombre vivía entonces en la ignorancia más completa de las leyes económicas y políticas que determinan el auge, desarrollo o muerte de los núcleos poblados.

La historia de la civilización humana nos enseña que los primeros núcleos poblados se edificaron con fines de defensa, ya sea de los animales feroces y del animal más feroz que era el hombre.

En las guerras permanentes en que vivían los diferentes grupos humanos era de rigor pasar por el cuchillo toda la población de una ciudad o pueblo conquistado.

En el desarrollo de la cultura con la subdivisión del trabajo humano se generaron de pronto diferentes núcleos poblados, si atendemos y clasificamos su función económica territorial. A la aldea le corresponde el rola de residencia de hombres ocupados en la extracción de productos naturales o materias primas. 
Al pueblo agregado comúnmente a una aldea le corresponde la tarea de distribuir la producción, tanto de los productos naturales hacia los centros de elaboración, como de los productos elaborados hacia sus puntos de consumo.

La ciudad que siembre absorbe la función de pueblo, amplía la función económica de este a la elaboración ya sea manual de las artes u oficios o a la industria mecanizada, que, como tal, existe prácticamente desde la invención del vapor, y de la electricidad, puestas al servicio del hombre.

La metrópoli es la ciudad que por su situación geográfica atiende el comercio internacional de un sector económico, y en ella se operan todas las manifestaciones del intercambio intelectual, artístico, científico y político que ligan a los diferentes países y continentes.

Aldeas, pueblos, ciudades y metrópolis obedecen a la misma ley natural, que determinan el auge, desarrollo y muerte de los humanos. El tiempo que sólo existe donde hay velocidad y movimiento, es el que hace evolucionar y envejecer los seres biológicos como los núcleos poblados.

La ley a que obedece la evolución de los núcleos poblados es la misma que la de gravitación universal, que se establece en este orden de cosas.

Se formula diciendo que la necesidad de núcleos poblados, en sus diversas funciones económicas, sociales y culturales, se establece por la riqueza de un territorio, y de que esta función económica, social y educativa se concentra en razón inversa al cuadrado del incremento de las velocidades del transporte y movilización.

La importancia enorme que el conocimiento de este postulado tiene para los administradores de pueblos y ciudades y políticos dirigentes, nos induce a detenernos en un análisis de este problema.

Imaginemos el caso utópico que en el transcurso de un año, por algún invento extraordinario, todos los medios de locomoción desarrollan el doble de velocidad. Esto significaría que todos los recorridos podrían operarse en la mitad del tiempo.

Con esto se concentrarían los almacenes de depósitos de mercaderías, casas de comercio mayorista, bancos, cajas, juzgados, Tesorerías, Oficinas públicas y escuelas en los centros poblados más favorecidos por la vialidad territorial, y como consecuencia los núcleos poblados de intercambio y elaboración se reducirían numéricamente a la cuarta parte.

Este fenómeno se opera en la práctica, ya que somos testigos del aumento de velocidad que permanentemente se opera en los medios de locomoción.

Siempre que este aumento de la velocidad fuera proporcional con el aumento de la riqueza territorial, medida en sus índices de extracción y elaboración, se mantendría el equilibrio en las funciones económicas de los núcleos poblados, pero hasta la fecha y 
particularmente en territorios de colonización el aumento de la velocidad ha sido muy superior al incremento de la riqueza territorial.

Las previsiones y consecuencias de este fenómeno de concentración de funciones económicas, debe tomarse en cuenta en el caso de Magallanes y se deducen de un análisis de las posibilidades de incremento de la riqueza y del mejoramiento de la vialidad territorial.

Analizando el primer aspecto del problema, anotamos la tendencia actual a establecer los centros de elaboración en el mismo lugar de la extracción con lo que se ahorra en transporte superfluo.

En el negocio de la lana se ve la posibilidad económica de establecer plantas de lavado de lana, en la elaboración de lanolina.

En los frigoríficos se ve la posibilidad de establecer una gran industria de subproductos elaborados, ya sea de abonos, cuerdas, productos químicos, biológicos, y alimenticios de diversa índole.

El establecimiento de hilanderías no estaría fuera de las posibilidades de incremento de la función económica de Magallanes. En todo caso, la investigación de este problema debe hacerse con el concurso de economistas preparados.

Las posibilidades de la existencia del petróleo y nuevos métodos y usos que la técnica moderna ha encontrado para carbones jóvenes como los del Territorio, permiten en todo caso abrigar cierto optimismo al avaluar las posibilidades de desenvolvimiento económico futuro de la ciudad de Magallanes.

En lo referente a la vialidad territorial, así como la apertura del canal de Panamá, desvió una importante corriente de tránsito hacia esa nueva ruta, la apertura del Istmo de Ofqui acercará a Magallanes a extensos lugares de extracción, permitiendo el acceso de numerosas embarcaciones menores. La línea de aeronavegación es otro factor de incremento vial favorable a las actividades de orden financiero y determinará seguramente una mayor concentración de funciones económicas en el pueblo, lo que a su vez permitiría el establecimiento de nuevas funciones de orden económico, social o cultural, derivadas.

En cuanto a la categoría de núcleo poblado, que es o debe ser Magallanes, si en la actualidad sólo puede ser calificado como pueblo, por no ser centro de elaboración, alcanza por el extenso radio de su zona de extracción la calificación de ciudad al analizar el problema bajo otro punto de vista.

En todo caso le corresponde desarrollar a Magallanes diversas funciones que también le corresponden a una ciudad, a saber la de centro administrativo, social, educativo, financiero y de recreación, además de centro de almacenamiento y de distribución que le corresponde como pueblo. 


\section{1.- La zonificación de los sectores urbanos}

La zonificación de los sectores urbanos, el principio de toda acción urbanística, debe considerar por lo tanto en Magallanes; las siguientes zonas.

I. Zona Comercial, Administrativa, Cultural y de Recreación.

II. Zona de Bodegas, Barracas, Talleres, Garajes y Fábricas.

III. Zona de vivienda mínima popular que por el tamaño de la forma de edificar no puede mezclarse con edificación residencial de otra categoría.

IV. Zona residencial de edificación.

V. Zona residencial de edificación aislada.

VI. Zona de huertos obreros u hortícolas de abastecimiento parcial urbano.

\section{2.-Futura extensión y crecimiento}

Establecida esta zonificación deducida de las condiciones de la edificación existente, la tarea más difícil para el urbanista es la de dimensionar y establecer una previsión certera de las posibilidades de desenvolvimiento futuro de cada una de estas zonas urbanas. Del desarrollo y disposición de estas zonas de destino económico, que ya son centros de trabajo o centros de residencia, dependen las características del tránsito de la ciudad futura, cuyo desenvolvimiento ya puede ser eficaz o estar entrabado al estar mal dispuesta esta zonificación.

En todo caso la zonificación de destino económico debería hacerse en base de estadísticas de todas las actividades del pueblo de Magallanes.

\section{3.-Aspectos de la vida vegetativa}

Una ciudad respira, crece o decrece, se nutre y evacua en la misma forma que cualquier ser organizado. La renovación celular equivale a la reconstrucción de edificios ruinosos, y los fenómenos de la circulación también los observamos en la red de calles que por su constitución son iguales a la red de arterias y venas de un ser organizado. El reglamento de edificación local es el único medio de evitar anomalías en el funcionalismo vegetativo urbano.

Haremos un análisis somero de estas funciones vegetativas y de las disposiciones correspondientes que deberían existir en una Ordenanza Local de Edificación.

\section{Respiración}

El grado de higiene de una ciudad y de la cual dependen directamente los índices porcentuales de mortalidad, depende de la densidad de población en los distintos sectores urbanos. En un plano de densidad del pueblo de Magallanes hemos anotado sectores de una población superior a 200 habitantes por hectárea, sector que al tener edificación dominante de un piso, podría ser calificada de insalubre por esta razón. 
Las ordenanzas locales de edificación establecen en tales casos limitaciones a la superficie predial edificable, relacionada con la altura de la edificación, dentro de los sistemas de edificación, ya sea, cerrada o compacta, mixta o escalonada, ya aislada.

Estas limitaciones dispuestas en un porcentaje variable fija la densidad máxima en cada sector de destino económico diferente.

La densidad admisible, de acuerdo con los principios de higiene universalmente adoptados, es la siguiente:

- Sectores comerciales e industriales de edificación cerrada de 1 y 2 pisos, 200 habitantes; de 2 y 3 pisos hasta 300 habitantes por hectárea.

- Sectores residenciales populares en un piso de 80 a 120 habitantes, de 2 pisos hasta 150 habitantes por hectárea.

- Habitaciones residenciales aisladas, de 60 a 100 habitantes por hectárea.

\section{Áreas verdes}

Asegurada la pureza del aire por disposiciones que limitan la densidad de población por hectárea, corresponde proteger los sectores comerciales y residenciales de las posibles emanaciones del sector de elaboración, y de sus ruidos que pueden ser molestos para otros sectores urbanos.

Esto se obtiene con la eficacia máxima al interponer entre el sector de elaboración y los otros sectores urbanos una área libre de edificación, ya sea o no arbolada; esta área ya puede ser campo de deporte, un parque o un vivero de árboles, como también un sector de explotación del suelo o subsuelo con un destino industrial no molesto.

Aparte de esto, a las áreas verdes les corresponde más bien una tarea de propender a la creación urbana, aparte de que un sistema continuo de áreas verdes asegura mejor la renovación y pureza del aire de una ciudad.

En el diseño de vías para el futuro desarrollo urbano corresponde disponer en la amplitud debida, las áreas verdes de recreación y aislamiento correspondiente a cada zona de destino urbano.

\section{Crecimiento}

En una ciudad observamos a semejanza de los seres orgánicos dos clases de crecimiento, el crecimiento de extensión volumétrico que corresponde a la edad del desarrollo de un ser núcleo poblado, y la renovación celular que en los humanos se opera totalmente en 7 años y que en una ciudad debería operarse cada 50 años en la renovación de los edificios. 
En un hecho innegable que cada época y cada generación forma y plasma estructuras arquitectónicas relacionadas con el proceso material y cultural alcanzado en ella, de modo que edificios de más de cincuenta años de edad, pronto aparecen anacrónicos y anticuados.

En las ciudades del sur y en Magallanes en especial, ya se opera el cambio de la antigua edificación de madera por edificios de material sólido, y aún en ellos se operará la segunda etapa de renovación que reemplaza las estructuras de albañilería por estructura rígida de concreto armado.

Por esto, para la edificación de servicios públicos especiales, expuestos a evolución radical de métodos de trabajo como escuelas, propiciamos en todo caso que se evite edificaciones demasiado estables y que no se extienda su duración más allá de treinta o cuarenta años.

En cuanto al crecimiento de extensión, este busca en toda ciudad las primeras aguas y el aire puro, por lo que se puede anotar que por lo general una ciudad busca la altura, en sentido contrario a los vientos reinantes.

Las condiciones especiales de Magallanes y su situación geográfica con un viento permanente del sur de una intensidad que llega a ser molesta, indican que tanto en la ubicación y trazado de la nueva extensión deberá considerarse en forma especial la protección de las casas contra las molestias del viento.

\section{Circulación}

La circulación en las ciudades del sur, dispuestas con una amplitud inconveniente para la economía urbana y mucho más allá de las necesidades del tránsito que pueda originarse en muchísimos años, no ofrece, por lo general ningún problema que requiera una solución inmediata.

El plano de Puerto Montt, por ejemplo, con manzanas cuadradas de cincuenta metros y calles de veinte metros, sólo se justifica como medida de protección de viviendas de madera contra incendios.

En la segunda etapa de edificación de pueblo con edificios de material sólido hay un interés evidente de la ciudad para clausurar calle por medio, buscando con esta medida protección contra el viento sur y manzanas mejor conformadas.

Las manzanas cuadradas de cien metros en el pueblo de Magallanes permiten también la clausura de algunas calles sin edificación definitiva que desemboquen al mar, donde propiciamos a priori la disposición de una amplia avenida costanera.

Esta sugerencia tiene base en el hecho ya operado en la ciudad de Tomé, cuyo plano regulador consideró la clausura de dos calles, cuya venta dejó una utilidad de más de cien mil pesos al Municipio. 
Focos de congestión de tránsito sólo hemos encontrado hasta la fecha en ciudades sureñas frente a las estaciones del Ferrocarril, frente a los Mercados o en las cabeceras de puentes de alguna importancia.

También hay casos de congestión cuando un pueblo o ciudad es el paso obligado de un camino interurbano, por falta de caminos de circunvalación.

Una mala disposición de los mataderos obliga también en muchos casos a los animales a atravesar determinados sectores urbanos.

Otro fenómeno de importancia y muy molesto para el tránsito de una ciudad es el que se produce cundo la extensión urbana, no dirigida de acuerdo con planos reguladores científicamente concebidos, se opera a lo largo de la red caminera intercomunal, transformando el camino en calle con el perjuicio más grave para la vialidad interurbana y urbana.

En tales casos el plano regulador ya bien dispone a lo largo de los caminos de acceso fajas reservadas al cultivo agrícola, sin utilización posible de carácter urbano, o, en su defecto, exige de sus urbanizadores, que quieran formar poblaciones con frente a los caminos, a construir calles paralelas a lo largo de éstas, de manera que se llega a obtener perfiles de vías con tres calzadas, una central de alta velocidad a larga distancia y dos laterales para el tránsito local.

\section{Nutrición o abastecimiento}

En urbanismo el problema de abastecimiento es un problema de buena distribución de sus centros en la ciudad.

Comienza en disponer las ferias mayoristas en las redes de tránsito pesado y sus cruces con otras vías ya sean marítimas o fluviales.

Los mercados municipales que no tengan carácter de feria, por lo general son un factor de control de comercio minorista de artículos de primera necesidad.

Las ferias libres son el mejor medio para poner en contacto directo al productor con el consumidor eliminando a los intermediarios.

Los mercados y ferias de comercio minorista deben ubicarse en contacto de los sectores de vivienda popular exclusiva, con la previsión necesaria para el futuro con espacios libres antepuestos de desahogo para el tránsito futuro.

En los sectores residenciales exclusivos también corresponde disponer núcleos o subcentros comerciales de abastecimiento parcial, con edificación continua más compacta, con la posibilidad del establecimiento de artesanos, como gasfíteres, electricistas, zapateros, sastres, etc., de los cuales hay necesidad en todo sector residencial, sin que exista propiamente necesidad de que éstos se concentren exclusivamente en los sectores comerciales exclusivos. 
En el plano de la futura extensión urbana es indispensable una previsión ordenada de estas necesidades, evitando la promiscuidad antiestética de casas de residencia tipo chalet con almacenes de edificación continua con muros medianeros visibles.

\section{Evacuación}

Igual al fenómeno de digestión, una ciudad elimina permanentemente desperdicios de toda naturaleza, ya sean éstos de carácter orgánico o inorgánico.

El tratamiento industrial de estos residuos ha hecho progresos enormes, aprovechándose los del alcantarillado para obtener abonos de primer orden, como también de las basuras que pueden ser tratadas por fermentación o por cremación.

Consideramos que este problema queda fuera del marco de materias que pensamos divulgar, por lo que no nos detendremos mayormente en él.

\section{4.-Otras actividades urbanas}

\section{La recreación y estímulo}

Dentro de las necesidades humanas, los sociólogos han atribuido a la recreación y estímulo la misma importancia porcentual que el alimento, vestuario y vivienda. Por esto el alcoholismo será un vicio obligado en nuestras clases populares, mientras no estén a su alcance otros medios de recreación y de estímulo. El deporte, el teatro, la lectura, la conferencia de índole cultural, la música clásica, la belleza de las artes plásticas, pueden llegar al conocimiento de espíritus vírgenes de impresiones artísticas y estímulos de orden superior, a través de museos de especies y reproducciones de obras famosas, por medio del fomento del teatro obrero aficionado, de asociaciones musicales de canto o de instrumentos de viento o cuerda, de gimnasia rítmica, etc.

Ciudades europeas pequeñas, como Salzburgo, en Austria, cuentan con dos mil asociaciones musicales.

La labor educativa que a los Municipios les corresponde desarrollar a este respecto es enorme, encontrando en Magallanes un campo especialmente propicio por la influencia cultural que evidentemente ha ejercido el elemento extranjero en esta ciudad.

La manera más práctica de realizar esta obra es la construcción de "casas del pueblo" con locales e instalaciones para conferencias, exposiciones, representaciones, ensayos musicales, etc., edificios a cuya erección seguramente contribuiría la Caja de Seguro Obligatorio.

\section{Recreación para turistas y extranjeros}

Las posibilidades de Magallanes como centro de turismo dependen en gran parte de la recreación y estímulo que el turista pueda encontrar en el pueblo de Magallanes. 
Debemos considerar en este caso tanto al estanciero como al turista nacional o extranjero que se resuelve a emprender un viaje a tanta distancia, que por su naturaleza obliga a algunos días de permanencia en Magallanes.

La corta temporada de turismo nos hace pensar en la posibilidad de aprovechamiento de un casino de turistas construido en forma rústica como caso de entretenciones populares de invierno. Entra aquí la posibilidad de una cancha de patinaje junto a una piscina temperada, sala de conferencias y de concierto, un verdadero club de carácter sui géneris y de aprovechamiento múltiple.

En verano, con una decoración teatral adecuada, un local de esta naturaleza puede transformarse fácilmente en un casino con cabaret para turistas.

Otra necesidad que en si, constituiría un importante problema de propaganda nacional sería la construcción de un gran hotel de turistas, que como el Hotel de Arica podría ser un contraste notable con edificaciones de las regiones adyacentes y, por lo tanto, un símbolo del predominio cultural de Magallanes.

\section{El problema económico}

La comprensión de la facilidad y posibilidades económicas de los planos reguladores es indispensable para juzgar la practicabilidad de las proposiciones y estudios que constituyen un proyecto de esta naturaleza.

Ante todo hay que tener presente que no se trata de proyectos de realización inmediata, sino de programas técnicos definidos cuya realización se efectuará sumando el esfuerzo e iniciativa particular, coordinados a través de estos planos reguladores, en una acción común eficiente en beneficio de la colectividad.

El sistema de la alineación paulatina a medida que los edificios existentes se reedifican puede conformar una calle en el plazo de 20 años más o menos, tiempo relativamente corto si se considera la vida de una ciudad.

Tanto la Ley 4563 y Ordenanza General de Construcciones como el Decreto con Fuerza de Ley N0345, de 30 de mayo de 1931, sobre la misma materia, establecen por otra parte nuevas normas para la expropiación de propiedades, partiendo de la base que toda transformación urbana produce en el predio donde se efectúe una expropiación parcial, un mayor valor en el retazo restante, mayor valor o plusvalía que puede ser descontada del monto de la expropiación.

Considerando, además, que ya es una convención universalmente aceptada por todos los peritos tasadores que los predios expropiados disminuyen de valor en su fondo, por lo cual se estima el valor de éste en la expropiación y no el valor del terreno en el frente, puede decirse que hoy en día, en la aplicación del sistema de substracción de la plusvalía a los predios expropiados más una tasación técnicamente correcta, permite no sólo efectuar expropiaciones y ensanches de calles por alineación sino también por una acción rápida total. 
El sistema de explotación del mayor valor operado en los predios, para una obra importante urbana, ha sido empleado en Chile por primera vez en el camino a San Bernardo, donde se dispusieron fajas de tributación por metro cuadrado paralelas al nuevo camino. Llamamos sin embargo la atención de que este sistema habría sido eficiente no para un camino, sino para una calle, y este sistema de financiamiento fue el que obligó a la urbanización a lo largo del camino, perdiendo éste su carácter de vía de alta velocidad con grave perjuicio para el pueblo de San Bernardo.

En urbanismo no se recomienda ninguna obra urbana que no sea en tal forma reductiva que el mayor valor que se opera pague con exceso todas las obras de urbanización.

No es justo destinar dineros de la colectividad y de procedencia diversa en obras de carácter local y con injustos los cargos que se hacen al Ejecutivo cuando se dice que éste nunca ha hecho nada para una ciudad.

Las obras que ejecuta el Ejecutivo sólo pueden ser de interés más general, como ferrocarriles, puentes, tranques, etc., obras que en todo caso sólo se emprenden al existir una reproductividad económica local para el futuro.

Las obras de agua potable y alcantarillado deberían ser empresas municipales, y en Chile se ha hecho cargo el Ejecutivo de ellas por incapacidad económica de los Municipios para emprenderlas. Pero en todo caso el pago de estas obras a lo largo lo efectúa el vecindario local.

El pavimento también se financia actualmente gravando los predios que lo enfrentan con un monto unitario correspondiente al frente que ocupan.

La única función económica que debe existir es la del crédito que fácilmente lo obtiene una ciudad cuando existe una reproductividad efectiva de las obras que se emprendan. Llamó por eso bastante la atención del infrascrito las observaciones que se formularon a ciertas recomendaciones de carácter general sobre el plano de Magallanes en que proponíamos la construcción de una avenida costanera.

La Dirección de Obras Municipales hizo un presupuesto aproximativo rechazando la idea por su costo elevado. Sólo la incomprensión del problema puede ser origen de observaciones de esta naturaleza. En la Comuna de Providencia, de una población reducida y con un Municipio pobrísimo, donde el plano de transformación ya se realiza desde hace cuatro años, la iniciativa privada debe haber invertido en obras de urbanización varias veces el valor presupuestado para la construcción de la costanera de Magallanes.

Esta suma presupuestada nos parece por otra parte sumamente baja si nos proponemos realizar el trabajo dentro de un plan de 20 años. Estableciendo los métodos de expropiación y urbanización anotados por sectores, en que se opere una valorización real, este trabajo puede realizarse sin costo o gasto extraordinario alguno para el Municipio. 


\section{Aspectos estéticos}

Todos los aspectos de índole económica, financiera, social y técnica en los planos reguladores deben relacionarse y converger en una acción única armónica, la que en sí constituye el arte del urbanismo.

La necesidad de proteger la ciudad contra el viento sur puede ser origen de diferencias reglamentarias para la altura de la edificación, que puede originar efectos plásticos y estéticos interesantes para la ciudad.

La necesidad de ante-plazas frente a servicios colectivos, y la construcción de éstos, debe dar origen a disposiciones reglamentarias que cierren la edificación alrededor de estos recintos y con alturas máximas y mínimas relacionadas con los espacios libres antepuestos.

La edificación no puede tener un valor estético aislado, puesto que su belleza se percibe dentro del emplazamiento al lado de otros edificios.

El valor estético individual de un edificio se anula completamente si la edificación colindante, de carácter definitivo, no armoniza con éste, tanto en valor, altura, proporción, tendencias arquitectónicas y en todo decorativo.

Así como el individualismo económico ha hecho época y sólo su supervivencia y agonía es el origen de la crisis económica que vivimos, el individualismo en el orden arquitectónico cede a un colectivismo artístico sano, exponente de la cultura de nuestra época, que construye ciudades cómodas, sanas y bellas.

Hagamos una ordenanza local de edificación para Magallanes, dentro de esta tendencia en que cada edificio no sea más que una parte que formará el gran edificio colectivo de la ciudad, exponente de su cultura y progreso.

N. de R.:

-Fuente de este escrito: Revista "ARQUITECTURA" N6, Ed. Antares, abril de 1936, pp. 35 a 38.

-Conceptos, lugares, instituciones, personas, citados:

Magallanes. Acrópolis. Pompeya. Aldea. Pueblo. Ciudad. Metrópolis. Ley de gravitación universal. Concentración de funciones económicas. Canal de Panamá. Istmo de Ofqui. Zonificación de sectores urbanos (Magallanes). Vida vegetativa. Densidad de población en sectores urbanos. Ordenanzas locales de edificación. Renovación de edificación (ciclos). Puerto Montt. Caminos interurbanos. Caminos de circunvalación. Abastecimiento. Evacuación. Salzburgo, Austria. Caja de Seguro Obligatorio. "Casas de Pueblo". Hotel de Arica. Ley 4563. D.F.L. N0345. San Bernardo. Avenida Costanera (Magallanes). Comuna de Providencia. 